\title{
Control de infecciones por Stapbylococcus aureus resistente a meticilina en una unidad de neonatología
}

\author{
María Angélica Oto L. ${ }^{1}$; María Eugenia Pinto $\mathrm{C}^{2}$; Virginia Martinez C. ${ }^{3}$; \\ Celia Fabio B. ${ }^{4}$ : Margarita Soza A. ${ }^{5}$; Raquel Jerez A. ${ }^{4}$; \\ Beatriz Garay G.6
}

\section{Control of methicillin-resistant Stapbylococcus aureus at a neonatal ward}

\begin{abstract}
Control measures for a methicillin-resıstant Staphylococcus aureus epidemic outbreak, in a neonatal unit of a metropolitan general hospital at Santiago, are described along with pertinent results. Control measures, including personnel education, attention to handwashing techniques, strict isolation of all infected and colonized cases, prospective microbiological surveillance of newborns, healthcare workers and environment, togetther with erradication of the agent from patients and personnel, were applied along a four month period and compared with the previous four months. Colonization rates and the incidence of diseases caused by methicilin-resistant Staphylococcus aureus decreased from 30.7 to $16 \%$ and 12.6 to $6.8 \%$, respectively $\{p<0.05$ )
\end{abstract}

(Key words: Staphyfococcus aureus, methicillin-resistant, neonatal infections, prevention.)

En las unidades de neonatologia la incidencia de las infecciones bacterianas varia en relación a los porcentajes de recién nacidos $(\mathrm{RN})$ de bajo peso de nacimiento (menores de $2500 \mathrm{~g}$ ) y de muy bajo peso de nacimiento (menores de $1500 \mathrm{~g}$ ), los recursos humanos y materiales dis. ponibles para su atención y el cumplimiento de las técnicas de enfermería.

Las infecciones bacterianas neonatales tradicionalmente se clasifican, según su gravedad, en

1. Departamento de Pediatría y Cingía Infantil Facultad de Medicina, División Ciencias Médicas Occidente, Universidad de Chile. Unidad de NeonatoJogja, Servicio de Pediatria, Hospital San Juan de Dios.

2. Unidad de Microbiología. Facultad de Medicina, División Ciencias Médicas Occidente, Universidad de Chile. Hospital San Juan de Dios.

3. Unidad de Anatomía Patológica, Facultad đe Medicina, División Ciencias Médicas Occidente, Universidad de Chile. Hospital San Juan de Dios.

4. Enfermera. Unidad de Neonatología, Servicio de Pediatría, Hospital San Juan de Dios.

5. Enfermera. Infección Intrahospitalaria, Hospital San Juan de Dios.

6. Tecnólogo Médico. Labontorio de Microbiología, Hospital San Juan de Dtos. mayores y menores. Las mayores incluyen septicemia, bronconeumonia, meningitis, osteoartritis, enterocolitis necrótica, infección del tracto urinario y diarrea aguda. Entre las infecciones localizadas o menores están la conjuntivitis, onfalitis, rinitis, impétigo e infección de herida operatoria. Otra clasificación se refiere al mo. mento de inicio de la infección, y se divide en precoces y tardias. Las precoces se manifiestan clínicamente en las primeras 48 horas de vida y se originan generalmente en una infección ovular o colonización matemas; y las tardias aparecen después de las 48 horas de vida, y habitualmente son de origen intrahospitalario.

Staphylococcus aureus resistente a meticilina (SAMR) apareció en Europa a fines de la década del 60 y en Norteamérica a fines de la década del 70 , como causa de infecciones graves intrahospitalarias. Constituyó un problema más extenso e importante en el decenio de los 80 , especialmente en grandes hospitales de nivel terciario y en aquellos en que se ejercía docencia $^{k}$. En Gran Bretaña SAMR aumentó de 1\% en 1978 a $28 \%$ en $1983^{2}$.

En la Unidad de Neonatologia del Hospital San Juan de Dios el principal microorganismo 
causal de las infecciones tardías, desde 1984, ha sido Staphylococcus aureus. Con el transcurso de los años ha aumentado la frecuencia de aislamiento de cepas que presentan un patrón de mayor resistencia, llegando SAMR a representar aproximadamente 50\% de los Staphylococcus aureus aislados en 1988. En febrero de ese año, de cada 30 nitos hospitalizados -como pro. medio diario-, 10 tenian infecciones por SAMR, que en cuatro de ellos eran graves (septicemia, bronconeumonía $y, u$, osteoartritis piógena) y en los otros seis localizadas.

Para intentar reducir la tasa de infección por SAMR se desarrolló un proyecto que incluía educación, establecimiento y aplicación de normas y apoyo microbiológico, cuyos resultados fueron evaluados conparando la incidencia de infección por SAMR con el periodo inmediatamente anterior y constituyen el propósito de esta comunicación.

\section{Pacientes y Método}

El estudio abarcó un período de ocho meses desde el 1 de diciembre de 1987 hasta el 31 de julio de 1988 , separado a su vez en dos periodos consecutivos de cuatro meses cada uno (períoda $I$ del 1 de diciembre de 1987 al 31 de marzo de 1988 y $\mathbf{~ I I ~ d e l ~} 1$ de abril al 31 de julio del mismo año). Se incluyeron todos los RN hospitalizados en la Unidad de Neonztología del Hospital San Juan de Dios, nacidos en la maternidad y trasladados de ot ros centros asistenciales. En el primer perjodo ingresaron a la unjdad de neonatología 237 nir̃os, 21 de los cuates tenían peso de nacimiento inferior a $1500 \mathrm{~g}$. En el segundo período se hospitalizaron 250 pacientes, 28 de los cuales correspondían a recién nacidos de muy bajo peso de nacimiento. El período I precedió a las medidas de control de infección y el II corresponde a aquel en que se tomaron las medidas detalladas más adelante. En el æegundo período se dispuso de material desechable suficiente para el cumplimiento adecuado de las técnicas de enfermería. Se calcularon tasas de incidencia promedio por períado de cuatro meses, constituyendo el numerador el número de infecciones por SAMR y el denominador el número de pacientes egresados en ese período de tiempo. Se consideró también el tipo de infección y su letalidad.

Las medidas de control del brote epidémico que se tomaron en el período II incluyeron información y motivación de personal; confección de normas de ingreso y flujo de circulación de los niños en la unidad; control microbiológico: erradicación del agente etiológico local y general y reforzamiento de las técnicas de aseo. En cumplímiento de lo anterior se informó de este proyecto a todo el personal de la unidad, se aclararon las dudas y se motjvó, mediante charlas y demostraciones, especialmente en relación a técnicas de lavado de manos y de aislamjento.

La unidad de recién nacidos, que cuenta con capacidad para 42 pacientes, está constituilla por una sala de cuidado intensivo, cuatro de cuidado intermedio, una de cuidado intermedio-mínimo y una de cuidado mínimo. Durante el brote epidémico dos salas de cuidado intermedio fueron habilitadas como aislarniento en cohorte para SAMR, una destinada a cujdado intensivo Y la olfa a cuidado intermedio. En cuanto a las mormas de ingreso y flujo de círculación de los niños, los RN provenientes de la maternidad del Hospital y de otros centros asistenciales ingtesaron a salas ljbres de SAMR. A los RN que habían permanecido en UCI se les exigió un cultivo nasal negativo, antes de ser trasladados a sala de cuidados intermedios. Los RN en que se aisló SAMR como colonización o infección se trasladaron a las salas de SAMR para aislamiento en cohorte, con técnicas de aislamiento de contacto, que incluyeron uso de mascarilla, dclantal y guantes (figura).

Se realizó cultivo nasal a los RN hospitalizados en las primeras 72 horas de su ingreso, a los RN considerados contactos de niños colonizados o infectados y en los pacientes de UCl antes de su traslado a sala de intermedio de flujo de UCI. También se hizo control microbiológico después del intento de erradicación del

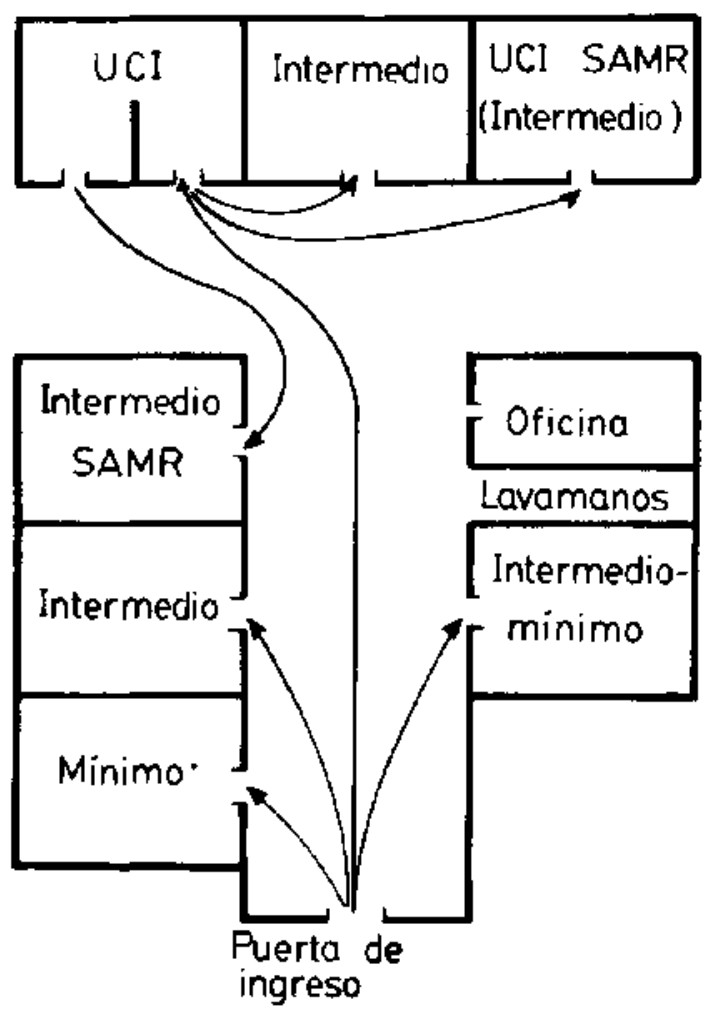

Figurn: Flujograma Unidad đe Neonatolog ía, Hospital San Juan de Dios. 
microorganismo con antinticrobianos tópicos. Tam bjén se efectuaron cultivos masales y de manos a todo el personal médico y paramédico de la unidad de neo. natologia; uno nasal y uno de manos en cos períodos: a 57 personas en abril-mayo y a 56 en julio de 1988 . Fi nalmente se tomaron muestras para cultivos de equipos y accesorios utilizados en la unidad de neonatología, pero solamente en el mes de junio de 1988.

La muestra endonasal se obtuvo mediante tórula estéril, rotándola en la superficie de la mucosa. El cultivo de las manos se realizó mediante impresión digital sobre placa de agar sangre. Las muestras para estudio microbiológico de superficie e instrumentos se lograron frotando sobre su superficje una tórula estéril humedecida en solución de $\mathrm{NaCl} \mathrm{9 \%} / \mathrm{oo}$. Las muestras de catéteres y tubos corrugados se recolectaron haciendo circular caldo cerebro-corazón por su interior. Todas las muestras obtenidas con tórula o caldos fueron sembradas en placas de agar sangre ( $5 \%$ ) y caldo thioglicolato e incubadas por 18 a 24 horas a $35^{\circ} \mathrm{C}$ en aerobiosis. La identificación posterior de las cepas bacterianas aisladas se realizó de acuerdo a las técnicas habituales ${ }^{3}$.

Se determinó la susceptibilidad de las cepas identificadas como Stophylococcus aureus a los siguientes antimicrobinanos: oxacilina, lincomicina, cotrimoxazol, cloramfenicol, tetraciclina, rifampicina y vancomicina, utilizando la técnica de Kirby-Bauer ${ }^{3}$. La susceptibili dad a oxacilina se realizó utilizando disco de $1 \mathrm{mcg}$ del antibiótico e incubar a $30^{\circ} \mathrm{C}^{3}$.

Para los efectos de este protocolo de estudio se definió infección como la presencia de microorganismos en los tejidos o fluidos corporales, acompañada de thanifestaciones clínicas adversas, locales o sistémicas, y se consideró colonización a la persistencia de microorganismos en la piel, mucosas o tejidos sin manifestaciones clínicas adversas ${ }^{4}$. Para hacer el control del crecimiento bacteriano se evaluó la eficacia de un ungüento tópico intranasal de neomicina y bacitracina para erradicar SAMR a nivel nasal en los niños cada 12 horas y pat 7 días. La erradicación de portación nasal del personal se realizó con el mismo ungüento e igual dosis, agregando trimetroprimsulfametoxazol por via oral en dosis de $160 \mathrm{mg}$ de trimetropim cada 12 horas por 7 días. En presencia de colonjzación de manos del personal se indicó lavado con clorhexina tres veces al día durante una semana. El personal portador de SAMR se asignó a la sala de aislamiento. Una vez diagnosticada la infección local o general, ésta se trató lo más precozmente posible. Las bronconeumonías, septicemias y osteoartritis piógenas a SAMR se trataron según su susceptibilidad in vitro, empleando lincomicina, fosfornicina, trimetroprimsulfametoxazol (en forma ocasional) y vancomicina (en un paciente solamente). Aun cuando se considera que vancomicjna es el antíbiótico de elección para infecciones graves a SAMR, sólo se dispuso de ella para uno de los RN infectados en 1988. La duración del tratamiento antimicrobiano en bronconeumonias y septicemias fluctuó entre 14 y 21 días $y$ en osteoartritis fue de 45 días. Cada 30 días se realizó aseo teminal de las salas de hospitalización y semanalmente se cambiaron los nitjos de las incubadoras, si los números de hospitalizados e incubadoras disponibles lo permitían. En el análisis estadístico de los resultados se empleó el método de chi cuadrado.

\section{Resultados}

En el período I se pesquisaron 30 infecciones por SAMR, $10(33 \%)$ de ellas eran infecciones mayores y 8 de éstas bronconeumonías. De las 20 infecciones menores, las más frecuentes fueron conjuntivitis y onfalitis. En consideración a estos hallazgos, en los últimos once días del mes de marzo se realizaron cultivos rasales de los RN hospitalizados con el fin de comprobar la tasa de colonización, confirmándose ésta en $16 / 52 \operatorname{casos}(30,7 \%)$.

En el segundo periodo, en el cual se establecieron y aplicaron las medidas de control, se comprobaron 17 infecciones, cuatro mayores y 13 menores. Se observó, por lo tanto, un des. censo de las bronconeumonías de ocho a dos, persistiendo igual número de septicemias (dos en cada período). Disminuyeron las onfalitis, manteniéndose el número de conjuntivitis. Al comparar el numero de infecciones mayores y menores en ambos períodos se comprobó una diferencia estadísticamente significativa ( $p<$ 0,05 ) (tabla). Diez pacientes presentaron infección por SAMR en dos localizaciones y tres en tres localizaciones diferentes, según diagnóstico clínico y documentación bacteriológica.

Table

Variación en tipo y frecuencia de infecciones por SAMR en dos periodos de 4 meses consecutivos, 1988

Fecha

Periodo I Período II

\begin{tabular}{|c|c|c|c|}
\hline Tasa por 100 egresos & $12,6 \%$ & $6,8 \%$ & \\
\hline $\begin{array}{l}\text { Lnfecciones mayores } \\
\text { Septicemia } \\
\text { Bronconeumonía } \\
\text { Subtotal }\end{array}$ & $\begin{array}{r}n \\
2 \\
8 \\
10\end{array}$ & $\begin{array}{l}\text { In } \\
2 \\
2 \\
4\end{array}$ & $\mathrm{p}$ \\
\hline $\begin{array}{l}\text { Infecciones menores } \\
\text { Conjuntivitis } \\
\text { Rinitis } \\
\text { Onfalitis } \\
\text { Impétigo } \\
\text { Herida operatoria } \\
\text { Subtotal }\end{array}$ & $\begin{array}{r}8 \\
4 \\
5 \\
2 \\
1 \\
20\end{array}$ & $\begin{array}{r}8 \\
2 \\
2 \\
1 \\
0 \\
13\end{array}$ & \\
\hline Total & $30 / 237$ & $17 / 250$ & $<0,05$ \\
\hline
\end{tabular}

Períado I: Periodo de referencia.

Perjodo II: Protocalo de control de infecciones. 
La tasa de colonización nasal por SAMR de los RN hospitalizados fue, en el segundo periodo, de $16 \%$ promedio $(48 / 299)$, lo que representa una disminución significativa con respecto al periodo anterior donde era $30,7 \%$ (p $<0,05$ ). En 29 de $42 \mathrm{RN}$ tratados con ungüento tópico intranasal de neomicina y bacitracina, se realizaron cultivos postratamiento, persistiendo positivos los aislamientos en $5 / 29$ $(17,2 \%)$.

Dos niños fallecieron debido a infecciones mayores por SAMR, $1 / 10$ y $1 / 4$ en el primer $y$ segundo periodo, respectivamente. En ambos pacientes el diagnóstico se confirmó en el estudio anatomopatológico. En el primer nifho se encontraron bronconeumonía, septicemia y osteoartritis piógena. En el segundo, bronconeumonia, septicemia, meningitis, miocarditis y abscesos renales.

Al iniciar el segundo período se confirmó aislamiento de SAMR en 4 de 57 personas (7\%) del equipo de salud estudiadas (dos enfermeras y dos auxiliares). En el segundo control, hecho con un intervalo de 3 meses, se comprobó portación en 2 de $56(3,6 \%)$ adultos (un médico y una kinesióloga). La erradicación, en estos seis adultos colonizados, se logró con tratamiento local y general. Al finalizar el segundo período el cultivo de seis equipos y accesorios (una incubadora de transporte, el sensor de dos oxímetros, un sensor de Doppler y dos mangueras corrugadas de un ventilador mecánico) dio resultado negativo para SAMR.

\section{Discusión}

En la Unidad de Neonatologia del Hospital San Juan de Dios se logró disminuir en forma significativa la colonjzación e infección por SAMR, al desarrollar y aplicar un programa de control del microorganismo durante cuatro meses (periodo II), a pesar de existir un número mayor de egresos y de $\mathrm{RN}$ con peso inferior a $1500 \mathrm{~g}$. Durante el segundo período se dispuso de insumos desechables en cantidad suficiente $y$ calidad adecuada. Experiencias similares se han realizado en adultos y RN, con buenos resultados. Para lograr estos resultados se requiere mantener una vigilancia epidemiológica y microbiológica activa y permanente ${ }^{5-8}$. Se ha podido documentar una relación entre el aumento de las infecciones en general, emergencia de cepas mul- tirresistentes y disninución de recursos humanos destinados a la atención de pacientes hospitalizados. Esto se observa especialmente en pacientes inmunodeprimidos, de unidades de cuidados intensivos, 0 ambos, sometidos a procedimientos y terapias invasivas, fundamentalmente ventilación mecánica a través de un tubo endotraqueal ${ }^{5,6,9-12}$.

SAMR tiene una capacidad patógena similar a $S$. aureus sensible a meticilina y con frecuencia causa septicemia, neumonia, osteomielitis y endocarditis bacteriana ${ }^{6,7}$, además de infecciones localizadas ${ }^{2,5}$. En nuestra experiencia fueron frecuentes las bronconeumonías y septicernias, $y$ entre las infecciones localizadas las conjuntivitis. Las colonizaciones suelen ser más frecuentes que las infecciones ${ }^{2}$, pero durante un brote epidémico la tasa de infección es alta ${ }^{2} . S$. aureus generalmente se disemina a través de las manos del personal $7,9,11,13$, de ahí la importancia de las técnicas adecuadas para el lavado de manos y el aislamiento local con delantal y guantes ${ }^{11}$. Estas dos medidas serían suficientes para prevenir la transmisión de SAMR durante los períodos endémicos, disminuyendo el costo de hospitalización y mejorando el cujdado del paciente ${ }^{11}$. El aislamiento en cohorte $y$ el uso de mascarilla estarían indicados solamente durante los brotes epidémicos ${ }^{3,9}$ y en pacientes sometidos a ventilación mecánica o que, estando traqueostomizados, eliminan secreción purulenta ${ }^{9}$. $S$. aureus nasal puede diseminarse por vía aéres si se agrega infección del tracto respiratorio superior ${ }^{10}$. Las manos también tendrían un rol en la diseminación a partir del reservorio nasal. Cuando se introduce $y$ establece SAMR en un hospital es muy difícil erradicarlo ${ }^{6,}{ }^{7}$. La portación puede prolongarse por meses, especialmente en los pacientes que han sufrido infección manifiesta ${ }^{6,}$ ? Para eliminarlo se requieren varias medidas simultáneas, una de ellas es la erradicación de por. tadores entre el personal. Durante un brote epidémico el personal puede constituir un reservorio importante que ayuda a la diseminación de SAMR en el hospital y en la comunidad ${ }^{14}$.

En el brote epidénico de nuestra unidad la tasa de portación fluctuó entre 3,6 y $7,0 \%$, cifras inferiores a las de un estudio norteamericano efectuado durante un período endémi$\mathrm{co}^{13}$. La erradicación de SAMR en nuestro personal, utilizando tratamiento local y general, se logró en todos los casos, con notable ahorro de morbilidad y coste ${ }^{s}$. 
Este estudio sirvió de modelo en nuestra unidad neonatal. Como resultado de su empleo continuado en 1991 se ha logrado la erradicación de SAMR, lo que parece guardar relación con la mantención de una disciplina de equipo en la prevención de la infección y el cumplimiento adecuado de las normas en la sala de atención inmediata de $\mathrm{RN}$ y en las de hospitalización.

Existen escasas publicaciones sobre brotes epidémicos por SAMR en unidades neonatales en el extranjero ${ }^{2,8}$. Esta experiencia nacional podría ser de utilidad en otros centros neonatales.

\section{Resumen}

Durante 4 meses de 1988, en la Unidad de Neonatología del Hospital San Juan de Dios, se realizó un proyecto de control de Staphylococcus aureus meticilina-resistente, y se compararon tasas de incidencia y tipo de infecciones en relación a un período anterior de 4 meses. Las medidas de control del brote epidémico incluyeron: información y motivación del personal especialmente en relación a técnica de lavado de manos y aislamiento, ordenamiento del ingreso y flujo de circulación de los niños en la unidad, aplicación de técnicas de aislamiento estricto en colonizados e infectados, control microbjológico de los recién nacidos, personal y ambiente; y erradicación del agente etiológico con antibióticos tópicos y orales. En este periodo de cuatro meses, en comparación a los 4 meses anteriores, disminuyó significativamente la tasa de colonización e infección debida a Staphylococcus aureus resistente a meticilina de 30,7 a $16 \%$ y de 12,6 a $6,8 \%$, respectivamente. Con esta medida de control de infección intrahospitalaria realizada simultáneamente, fue posible disminuir en forma significativa la colonización $\mathrm{e}$ infección por Staphylococcus aureus resistente a meticilina.

(Palabras claves: Staphylococcus aureus, meticilina-resistente, recién nacidos, infección neonatal, intrahospitalaria, prevención, control.)

\section{Agradecimientos}

Se agradece a Colomba Norero $v$, su yaliosa colaboración en la revisión de este manuscrito.

\section{Referencias}

1. Haley $R W$, Hightower $A W$. Khabbaz $R F$ et al: The emergence of methicillin-resistant Staphylococus oureus infections in United States hospitals. Ann intern Med 1982;97: 297-308.

2. Parks YA, Noy MF. Sukett MA, Webb CA: Methicillin-resistant Staphylococcus aureus in milk. Arch Dis Child 1987;62: 82-84.

3. Kloos WE, Lambe DW: Staphylococcus En: Ballows A, Hausler WJ, Herrmann $K \mathrm{~L}$, Isenberg HD, Shadomy HJ. ed. Manual of Clinical Microbiology, Washington, DC: American Society for Microbiology. 5 th edition, $1991 ; 222-237$.

4. Ministerio de Salud. 1986 . Sistema de vigila ncia de infecciones intrahospitalarias y Manual de definiciones.

5. Rao $N$, Jacobs $S$, Joyce L: Cost-effective eradication of an outbreak of methicillin-resistant Sta. phylococcus aureus in a community teaching hospital. Infect Control Hosp Epidemiol 1988 ; 9: $255-260$.

6. Murray-Leisture KA, Geib $S$, Graceley $D$ et al. Control of epidemic methicillin-resistant Staphy. lococcus aureus. Infect Controt Hosp Epidemiol $1990 ; 11: 343-350$.

7. Guiguet $M$, Rekacewicz $C$, Leclercq $B$, Brun $Y$, Escudier B, Andremont A: Effectiveness of simple measures to control an outbreak of nosocomial methicillin-resistant Sraphylococcus aureus infections in an intensive care unit, lnfect Control Hosp Epidemiol 1990; 11: 23-26.

8. Dunkle LM, Naqui SH, McCalum $R$, Lofgren $J P$ : Eradication of epidemic methicillin-gentamicin-tesistant Staphylococcus aurews in an intensive care nursery. Am J Med 1981; 70: 455-458.

9. Kauffman $C A$, Bradley $S F$, Terpenning $M S$ : Methicillin-fesistant Staphylococcus aureus in longterm care facilities. Infect Control Hosp Epidemiol $1990 ; 11: 600-603$.

10. Beloni A, Shererti RJ, Sullivan ML, Russell BA, Reumon PD: Outbresk of Stophylococcus infection in two hospital nurseries traced to a single nasal canier. Infection Control 1986; 7: 487490.

11. Ribner BS, Landry $M N$, Gholson GL: Strict versus modified isolation for prevention of nosocomial transmission of methicillin-resistant Staphylococcus aureus. Infection Control 1986; 7: 317-320.

12. Rosenfeld ChR, Laptook AR, Jeffery I: Limited effectiveness of triple dye in preventing colonization with methicillin-resistant Staphylococcts aureus in special care nursery. Pediatr Infect Dis J 1990; 9: 290-291.

13. Opal SW, Mayer KH, Stenberg $M L$ ef al.: Frequent acquisition of multiple strains of methicillinresistant Staphylococcts aurews by healthcare workers in an endemic hospital environrnent. Infect Control Hosp Epidemiol 1990; 11: 479485 .

14. Reboli AC, John JF, Ptatt CG, Cantey JR: Methicillin-resistant Staphylococcus outbreak at a veterans affairs medical center: importance of carriage of the organism by hospital personnel. Infect Control Hosp Epidemiol 1990; 11: 291296. 